\title{
Fatty acid content and pattern of spleen phospholipids and triglycerides in normal and either type-1 or type- 2 diabetic rats
}

\author{
LAURENCE PORTOIS $^{1}$, ABDULLAH SENER ${ }^{2}$, YVON A. CARPENTIER ${ }^{1}$ and WILLY J. MALAISSE ${ }^{2}$ \\ Laboratories of ${ }^{1}$ Experimental Surgery and ${ }^{2}$ Experimental Hormonology, \\ Brussels Free University, B-1070 Brussels, Belgium
}

Received October 20, 2006; Accepted November 29, 2006

\begin{abstract}
The fatty acid pattern of spleen phospholipids and triglycerides was examined in fed or overnight fasted normal rats, streptozotocin-induced diabetic animals (type-1 diabetes) and Goto-Kakizaki rats (type-2 diabetes). In both phospholipids and triglycerides, differences were observed in the relative contribution of several fatty acids, as well as in the ratio between distinct fatty acids, when comparing fed to fasted rats, normal to diabetic animals and male to female Goto-Kakizaki rats. Diabetes increased to a greater extent the C22:6w3 content of phospholipids in the spleen than in either the liver or the brain. However, the diabetes-induced changes in the C22:6w3 content of triglycerides was closely comparable in the spleen, liver and brain. These findings suggest that the incorporation of fatty acids into triglycerides is controlled by comparable regulatory factor(s), e.g. insulinemia, in the spleen, liver and brain. In the case of phospholipids, however, an apparent adaptation to diabetic stress was more marked in the spleen than in the liver, and virtually absent in the brain. The proposed dichotomy in the environmental regulation of fatty acid synthesis and incorporation into phospholipids and triglycerides was further supported by distinct diabetesrelated changes in the apparent activity of $\Delta 9$-desaturase in these two classes of lipids.
\end{abstract}

\section{Introduction}

Recent studies have drawn attention to differences between normal and either type- 1 or type- 2 diabetic rats in terms of the fatty acid content and pattern, not solely of adipose tissue lipids (1), but also of plasma (2), liver (2) and brain (3) phospholipids and triglycerides. The results of the present study extend this knowledge to the phospholipids and triglycerides of the spleen, which participates in defence and immune processes.

Correspondence to: Professor W.J. Malaisse, Laboratory of Experimental Hormonology, Brussels Free University, 808 Route de Lennik, B-1070 Brussels, Belgium

E-mail: malaisse@ulb.ac.be

Key words: spleen, phospholipid and triglyceride fatty acid pattern, fasting, type- 1 diabetes, type- 2 diabetes

\section{Materials and methods}

The present study was conducted with four female normal rats given free access to food up to the time of sacrifice (fed normal rats), four overnight fasted female normal rats (fasted normal rats) and six fed female streptozotocin-induced diabetic animals (STZ rats). These animals were injected with streptozotocin 3 days before sacrifice. In addition, four male and four female Goto-Kakizaki rats were examined either in the fed state or after overnight fasting. The metabolic and hormonal status of these 22 rats is described elsewhere (1).

At sacrifice, a fragment of the spleen was examined for the fatty acid content and pattern of both phospholipids and triglycerides (4). Only those fatty acids which remained below the limit of detection in all samples are not listed in Tables I and III. The procedure used in this study was approved by the local Animal Experimentation Ethics Committee.

All results are presented as mean values $( \pm$ SEM or range of individual variations, whenever derived from only two measurements) together with the number of individual determinations (n). The statistical significance of differences between mean values was assessed by use of the Student's ttest or, whenever required, by covariance analysis.

\section{Results}

Phospholipids. The spleen phospholipid content, expressed as fatty acid equivalent, was comparable in the different groups of rats under consideration (Table I). The most abundant fatty acids found in spleen phospholipids were C20:4 $\omega 6$,

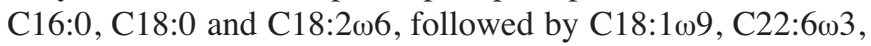
$\mathrm{C} 22: 5 \omega 3$ and $\mathrm{C} 22: 4 \omega 6$.

As a rule, the fractional contribution of each fatty acid was not vastly different in the several types of rats. Nevertheless, significant differences were often observed, as illustrated by the measurements of $\mathrm{C} 22: 6 \omega 3$. First, the weight percentage of $\mathrm{C} 22: 6 \omega 3$ was higher $(\mathrm{p}<0.01)$ in overnight fasted normal female rats $(4.29 \pm 0.22 ; n=4)$ than in fed normal female rats $(3.32 \pm 0.13 ; n=4)$. Variable to this finding, no difference between fed versus fasted animals was observed in either the male GK rats $(4.97 \pm 0.05$ versus $5.00 \pm 0.06)$ or female GK rats $(6.32 \pm 0.46$ versus $6.35 \pm 0.25)$. Second, as documented by the values just mentioned, an obvious gender difference prevailed in GK rats, with higher values $(\mathrm{p}<0.001)$ in female $(6.34 \pm 0.21 ; n=4)$ than male $(4.99 \pm 0.03 ; n=4)$ rats. Lastly, the 
Table I. Fatty acid content and pattern of spleen phospholipids.

\begin{tabular}{|c|c|c|c|c|c|c|c|}
\hline Rats & Fed normal (F) & Fasted normal $(\mathrm{F})$ & Fed STZ (F) & Fed GK (F) & Fasted GK (F) & Fed GK (M) & Fasted GK (M) \\
\hline Total (mg/g) & $11.4 \pm 0.4(4)$ & $11.0 \pm 0.4(4)$ & $11.4 \pm 0.3(6)$ & $10.6 \pm 0.3(2)$ & $10.3 \pm 0.6(2)$ & $11.1 \pm 0.7(2)$ & $12.2 \pm 0.4(2)$ \\
\hline $\mathrm{C} 14: 0(\%)$ & $0.3 \pm 0.0$ & $0.2 \pm 0.0$ & $0.2 \pm 0.0(6)$ & $0.2 \pm 0.0(2)$ & $0.2 \pm 0.0$ & $0.3 \pm 0.1(2)$ & $0.2 \pm 0.0(2)$ \\
\hline C16:0 (\%) & $25.3 \pm 0.5(4)$ & $26.2 \pm 0.4(4)$ & $26.8 \pm 0.2(6)$ & $23.5 \pm 0.2(2)$ & $24.3 \pm 0.1(2)$ & $25.6 \pm 0.7(2)$ & $25.4 \pm 0.2(2)$ \\
\hline $\mathrm{C} 16: 1 \omega 7(\%)$ & $0.4 \pm 0.0$ & $0.4 \pm 0.1$ & $0.3 \pm 0.0(6)$ & $0.3 \pm 0.0(2)$ & $0.5 \pm 0.1(2)$ & $0.4 \pm 0.1(2)$ & $0.3 \pm 0.0(2)$ \\
\hline C18:0 (\%) & $14.7 \pm 0.3$ & $15.1 \pm 0.3$ & $14.9 \pm 0.2(6)$ & $15.5 \pm 0.3(2)$ & $15.5 \pm 0.3(2)$ & $14.2 \pm 0.2(2)$ & $15.2 \pm 0.2(2)$ \\
\hline 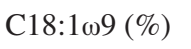 & $6.8 \pm 0.2$ & $5.8 \pm 0.1$ & $5.8 \pm 0.1(6)$ & $5.8 \pm 0.1$ & $6.4 \pm 0.2(2)$ & $5.4 \pm 0.1(2)$ & $5.7 \pm 0.1(2)$ \\
\hline $\mathrm{C} 18: 2 \omega 6(\%)$ & $14.2 \pm 0.4(4)$ & $10.7 \pm 0.2$ & $12.4 \pm 0.5(6)$ & $11.0 \pm 0.2(2)$ & $8.9 \pm 0.3(2)$ & $11.1 \pm 0.2(2)$ & $9.5 \pm 0.1(2)$ \\
\hline $\mathrm{C} 18: 3 \omega 6(\%)$ & $0.0 \pm 0.0$ & $0.0 \pm 0.0$ & $0.0 \pm 0.0$ & $0.1 \pm 0.1$ & $0.1 \pm 0.1(2)$ & $0.1 \pm 0.1(2)$ & $0.1 \pm 0.0(2)$ \\
\hline C20:0 (\%) & $0.3 \pm 0.0(4)$ & $0.3 \pm 0.0(4)$ & $0.3 \pm 0.0(6)$ & $0.3 \pm 0.1(2)$ & $0.2 \pm 0.0(2)$ & $0.3 \pm 0.0(2)$ & $0.3 \pm 0.0(2)$ \\
\hline $\mathrm{C} 18: 3 \omega 3(\%)$ & $0.1 \pm 0.0(4)$ & $0.0 \pm 0.0(4)$ & $0.1 \pm 0.0(6)$ & $0.0 \pm 0.0(2)$ & $0.0 \pm 0.0(2)$ & $0.1 \pm 0.1(2)$ & $0.0 \pm 0.0(2)$ \\
\hline 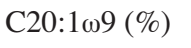 & $0.2 \pm 0.0$ & $0.2 \pm 0.0(4)$ & $0.2 \pm 0.0(6)$ & $0.2 \pm 0.0(2)$ & $0.2 \pm 0.0(2)$ & $0.2 \pm 0.0(2)$ & $0.2 \pm 0.0(2)$ \\
\hline 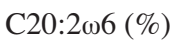 & $1.3 \pm 0.0(4)$ & $0.9 \pm 0.0(4)$ & $1.0 \pm 0.0(4)$ & $0.8 \pm 0.1(2)$ & $0.6 \pm 0.0(2)$ & $0.8 \pm 0.0(2)$ & $0.7 \pm 0.0(2)$ \\
\hline C22:0 (\%) & $0.4 \pm 0.0$ & $0.4 \pm 0.0$ & $0.5 \pm 0.0(6)$ & $0.5 \pm 0.1(2)$ & $0.4 \pm 0.0(2)$ & $0.4 \pm 0.0(2)$ & $0.5 \pm 0.0(2)$ \\
\hline 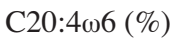 & $26.2 \pm 0.2$ & $28.7 \pm 0.3$ & $26.3 \pm 0.6(6)$ & $29.5 \pm 0.4(2)$ & $30.4 \pm 0.7$ (2) & $29.4 \pm 0.5(2)$ & $30.1 \pm 0.3(2)$ \\
\hline $\mathrm{C} 20: 5 \omega 3(\%)$ & $0.1 \pm 0.0$ & $0.1 \pm 0.0$ & $0.1 \pm 0.0$ & $0.2 \pm 0.1(2)$ & $0.1 \pm 0.1$ (2) & $0.1 \pm 0.1(2)$ & $0.1 \pm 0.1$ \\
\hline C24:0 (\%) & $1.0 \pm 0.1(4)$ & $1.0 \pm 0.0(4)$ & $1.1 \pm 0.0(6)$ & $1.0 \pm 0.1(2)$ & $1.0 \pm 0.1(2)$ & $1.0 \pm 0.1(2)$ & $1.1 \pm 0.1(2)$ \\
\hline $\mathrm{C} 22: 4 \omega 6(\%)$ & $2.8 \pm 0.1(4)$ & $2.4 \pm 0.1(4)$ & $2.4 \pm 0.1(6)$ & $2.6 \pm 0.1(2)$ & $2.6 \pm 0.2(2)$ & $2.8 \pm 0.0(2)$ & $2.9 \pm 0.1(2)$ \\
\hline $\mathrm{C} 22: 5 \omega 3(\%)$ & $2.3 \pm 0.1(4)$ & $3.3 \pm 0.1(4)$ & $3.1 \pm 0.1(6)$ & $2.6 \pm 0.0(2)$ & $2.6 \pm 0.2(2)$ & $3.2 \pm 0.1(2)$ & $3.1 \pm 0.2(2)$ \\
\hline $\mathrm{C} 22: 6 \omega 3(\%)$ & $3.3 \pm 0.1(4)$ & $4.3 \pm 0.2(4)$ & $4.6 \pm 0.3(6)$ & $6.3 \pm 0.5(2)$ & $6.4 \pm 0.3(2)$ & $5.0 \pm 0.1(2)$ & $5.0 \pm 0.1(2)$ \\
\hline
\end{tabular}

F, female; $M$, male.

Table II. Ratio between distinct fatty acids in spleen phospholipids.

\begin{tabular}{lccccccc}
\hline Rats & Fed normal (F) & Fasted normal (F) & Fed STZ (F) & Fed GK (F) & Fasted GK (F) & Fed GK (M) & Fasted GK (M) \\
\hline $\mathrm{C} 16: 0 / \mathrm{C} 16: 1 \omega 7$ & $64.2 \pm 3.9(4)$ & $62.4 \pm 5.2(3)$ & $85.9 \pm 2.7(6)$ & $72.3 \pm 3.0(2)$ & $53.5 \pm 1.4(2)$ & $74.3 \pm 2.2(2)$ & $91.4 \pm 5.5(2)$ \\
$\mathrm{C} 18: 0 / \mathrm{C} 18: 1 \omega 9$ & $2.17 \pm 0.08(4)$ & $2.62 \pm 0.09(4)$ & $2.59 \pm 0.04(6)$ & $2.66 \pm 0.09(2)$ & $2.42 \pm 0.10(2)$ & $2.65 \pm 0.07(2)$ & $2.70 \pm 0.01(2)$ \\
$(\mathrm{C} 16: 0+\mathrm{C} 16: 1 \omega 7) /$ & $1.20 \pm 0.03(4)$ & $1.28 \pm 0.01(4)$ & $1.31 \pm 0.03(6)$ & $1.12 \pm 0.02(2)$ & $1.13 \pm 0.00(2)$ & $1.32 \pm 0.03(2)$ & $1.23 \pm 0.02(2)$ \\
$(\mathrm{C} 18: 0+\mathrm{C} 18: 1 \omega 9)$ & & & & & & \\
& & & & & & \\
$\mathrm{C} 18: 2 \omega 6 / \mathrm{C} 18: 3 \omega 6$ & $192 \pm 12(2)$ & $149 \pm 2(2)$ & N.A. & $133(1)$ & $97(1)$ & $126(1)$ & $106 \pm 16(2)$ \\
$\mathrm{C} 18: 3 \omega 6 / \mathrm{C} 20: 4 \omega 6\left(\mathrm{x} 10^{3}\right)$ & $2.90 \pm 0.07(2)$ & $2.54 \pm 0.12(2)$ & N.A. & $2.80(1)$ & $2.85(1)$ & $2.97(1)$ & $3.02 \pm 0.47(2)$ \\
$\mathrm{C} 18: 2 \omega 6 / \mathrm{C} 20: 4 \omega 6$ & $0.54 \pm 0.02(4)$ & $0.37 \pm 0.01(4)$ & $0.47 \pm 0.03(6)$ & $0.37 \pm 0.00(2)$ & $0.29 \pm 0.01(2)$ & $0.38 \pm 0.00(2)$ & $0.31 \pm 0.00(2)$ \\
$\mathrm{C} 20: 4 \omega 6 / \mathrm{C} 22: 4 \omega 6$ & $9.47 \pm 0.24(4)$ & $11.87 \pm 0.34(4)$ & $11.01 \pm 0.26(6)$ & $11.25 \pm 0.32(2)$ & $12.06 \pm 0.56(2)$ & $10.37 \pm 0.13(2)$ & $10.60 \pm 0.23(2)$ \\
$\mathrm{C} 20: 5 \omega 3 / \mathrm{C} 22: 5 \omega 3\left(\mathrm{x} 10^{3}\right)$ & $45.9 \pm 2.9(3)$ & $37.1 \pm 15.4(3)$ & $73.2 \pm 14.7(4)$ & $55.5 \pm 25.9(2)$ & $86.2(1)$ & $56.6(1)$ & $45.7(1)$ \\
$\mathrm{C} 22: 5 \omega 3 / \mathrm{C} 22: 6 \omega 3$ & $0.83 \pm 0.04(4)$ & $0.78 \pm 0.03(4)$ & $0.69 \pm 0.03(6)$ & $0.42 \pm 0.03(2)$ & $0.40 \pm 0.00(2)$ & $0.65 \pm 0.02(2)$ & $0.62 \pm 0.04(2)$ \\
\hline
\end{tabular}

F, female; M, male; N.A., not applicable.

C22:6w3 relative content was significantly higher $(\mathrm{p}<0.005$ or less) in either STZ rats $(4.60 \pm 0.25 ; n=6)$ or GK rats $(6.32 \pm 0.46 ; n=2)$ than in normal rats $(3.32 \pm 0.13 ; n=4)$, all these results referring to fed female animals. Likewise, the weight percentage of $\mathrm{C} 22: 6 \omega 3$ was higher $(\mathrm{p}<0.005)$ in overnight fasted female GK rats $(6.35 \pm 0.25 ; n=2)$ than in overnight fasted normal rats $(4.29 \pm 0.22 ; n=4)$.

Further differences were observed in the paired ratios between distinct fatty acids (Table II). The C16:0/C16:1 107 paired ratio was not significantly different in fed versus fasted normal rats. Likewise, it was little affected by fasting in GK rats, with only a significant fasting-induced decrease $(\mathrm{p}<0.05)$ in female animals, the overall mean values averaging
$73.3 \pm 1.6$ and $72.5 \pm 11.2$ ( $n=4$ in both cases) in fed and fasted GK rats, respectively. The values found in fed GK rats represented $114.2 \pm 2.5 \%(n=4 ; p<0.08)$ of that found in fed normal rats $(100.0 \pm 6.0 \% ; n=4)$. Likewise, the $C 16: 0$ / $\mathrm{C} 16: 1 \omega 7$ paired ratio was significantly higher $(\mathrm{p}<0.005)$ in fed STZ rats than in fed normal rats. It was lower, albeit not significantly so, in female than male GK rats examined in the same nutritional status (fed or fasted).

The C18:0/C18:1 $\omega 9$ ratio averaged $2.17 \pm 0.08$ in normal female fed rats, and was increased ( $\mathrm{p}<0.025$ or less) to $2.62 \pm 0.09(n=4)$ in normal fasted rats, 2.59 $\pm 0.04(n=6)$ in STZ rats, $2.54 \pm 0.09(n=4)$ in female GK rats and $2.67 \pm 0.03$ $(n=4)$ in male GK rats. 
Table III. Fatty acid content and pattern of spleen triglycerides.

\begin{tabular}{|c|c|c|c|c|c|c|c|}
\hline Rats & Fed normal $(\mathrm{F})$ & Fasted normal (F) & Fed STZ (F) & Fed GK (F) & Fasted GK (F) & Fed GK (M) & Fasted GK (M) \\
\hline Total (mg/g) & $3.18 \pm 0.20(4)$ & $2.09 \pm 0.76(4)$ & $2.07 \pm 0.64(6)$ & $3.39 \pm 2.41(2)$ & $0.17 \pm 3.52(2)$ & $1.74 \pm 1.08(2)$ & $4.65 \pm 3.82(2)$ \\
\hline $\mathrm{C} 12: 0(\%)$ & $0.1 \pm 0.1(4)$ & $0.1 \pm 0.1(4)$ & $0.0 \pm 0.0(6)$ & $0.2 \pm 0.2(2)$ & $0.2 \pm 0.0(2)$ & $0.1 \pm 0.1(2)$ & $0.1 \pm 0.1(2)$ \\
\hline $\mathrm{C} 14: 0(\%)$ & $0.7 \pm 0.4(4)$ & $1.0 \pm 0.3(4)$ & $0.8 \pm 0.3(6)$ & $1.5 \pm 0.1(2)$ & $1.5 \pm 0.2(2)$ & $0.6 \pm 0.6(2)$ & $0.6 \pm 0.6(2)$ \\
\hline $\mathrm{C} 16: 0(\%)$ & $27.4 \pm 2.2(4)$ & $25.0 \pm 1.7$ & $25.9 \pm 0.5(6)$ & $25.5 \pm 1.3(2)$ & $25.5 \pm 0.1(2)$ & $23.0 \pm 0.3(2)$ & $21.8 \pm 0.1(2)$ \\
\hline $\mathrm{C} 16: 1 \omega 7(\%)$ & $1.8 \pm 1.1(4)$ & $2.7 \pm 0.6(4)$ & $2.1 \pm 0.8(6)$ & $4.4 \pm 1.1(2)$ & $5.4 \pm 0.7$ (2) & $2.4 \pm 0.1(2)$ & $1.8 \pm 0.3(2)$ \\
\hline C18:0 (\%) & $5.9 \pm 1.5(4)$ & $5.5 \pm 1.1(4)$ & $5.9 \pm 1.1(6)$ & $3.9 \pm 0.9(2)$ & $2.7 \pm 0.2(2)$ & $4.6 \pm 1.2(2)$ & $4.4 \pm 0.7(2)$ \\
\hline 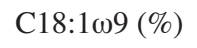 & $20.8 \pm 0.5$ & $21.6 \pm 0.9$ (4) & $19.3 \pm 1.1(6)$ & $23.8 \pm 0.2(2)$ & $25.7 \pm 0.3(2)$ & $21.2 \pm 0.5(2)$ & $23.6 \pm 0.6(2)$ \\
\hline $\mathrm{C} 18: 2 \omega 6(\%)$ & $39.7 \pm 1.9(4)$ & $41.3 \pm 2.0(4)$ & $38.3 \pm 2.0(6)$ & $34.2 \pm 0.5(2)$ & $33.6 \pm 1.4(2)$ & $40.1 \pm 2.5(2)$ & $41.9 \pm 0.1(2)$ \\
\hline $\mathrm{C} 18: 3 \omega 6(\%)$ & $0.1 \pm 0.1$ & $0.1 \pm 0.1$ & $0.0 \pm 0.0(6)$ & $0.2 \pm 0.2(2)$ & $0.2 \pm 0.0$ & $0.0 \pm 0.0$ & $0.1 \pm 0.1(2)$ \\
\hline $\mathrm{C} 18: 3 \omega 3(\%)$ & $2.4 \pm 0.8$ & $1.8 \pm 0.6(4)$ & $1.9 \pm 0.6(6)$ & $2.3 \pm 0.1(2)$ & $2.1 \pm 0.1(2)$ & $1.2 \pm 1.2(2)$ & $0.9 \pm 0.9(2)$ \\
\hline C20:1w9 (\%) & $0.1 \pm 0.1(4)$ & $0.1 \pm 0.1(4)$ & $0.0 \pm 0.0(6)$ & $0.0 \pm 0.0(2)$ & $0.1 \pm 0.1(2)$ & $0.0 \pm 0.0(2)$ & $0.2 \pm 0.2(2)$ \\
\hline $\mathrm{C} 20: 2 \omega 6(\%)$ & $0.0 \pm 0.0(4)$ & $0.0 \pm 0.0(4)$ & $0.0 \pm 0.0(6)$ & $0.2 \pm 0.2(2)$ & $0.3 \pm 0.1(2)$ & $0.0 \pm 0.0(2)$ & $0.2 \pm 0.2(2)$ \\
\hline C24:0 (\%) & $0.0 \pm 0.0$ & $0.0 \pm 0.0(4)$ & $0.0 \pm 0.0(6)$ & $0.4 \pm 0.4(2)$ & $0.1 \pm 0.1(2)$ & $1.3 \pm 1.0(2)$ & $0.8 \pm 0.8(2)$ \\
\hline $\mathrm{C} 22: 4 \omega 6(\%)$ & $0.2 \pm 0.1(4)$ & $0.1 \pm 0.1(4)$ & $0.8 \pm 0.5(6)$ & $0.9 \pm 0.5(2)$ & $0.4 \pm 0.1(2)$ & $1.7 \pm 0.9(2)$ & $1.6 \pm 0.9(2)$ \\
\hline $\mathrm{C} 22: 5 \omega 3(\%)$ & $0.2 \pm 0.1(4)$ & $0.2 \pm 0.1(4)$ & $1.3 \pm 0.7(6)$ & $0.9 \pm 0.4(2)$ & $0.4 \pm 0.0(2)$ & $1.9 \pm 0.8(2)$ & $1.6 \pm 0.9(2)$ \\
\hline $\mathrm{C} 22: 6 \omega 3(\%)$ & $0.4 \pm 0.2(4)$ & $0.4 \pm 0.2(4)$ & $2.9 \pm 1.4(6)$ & $1.9 \pm 0.3(2)$ & $1.1 \pm 0.0(2)$ & $2.2 \pm 0.7(2)$ & $0.5 \pm 0.5(2)$ \\
\hline
\end{tabular}

$\mathrm{F}$, female; $\mathrm{M}$, male.

The $(\mathrm{C} 16: 0+\mathrm{C} 16: 1 \omega 7) /(\mathrm{C} 18: 0+\mathrm{C} 18: 1 \omega 9)$ ratio was not affected significantly by fasting, with the results recorded in the overnight fasted rats averaging $101.6 \pm 2.1 \%(n=8)$ of the mean corresponding values $(100.0 \pm 1.3 \% ; n=8)$ found in fed rats of the same sex and strain. This ratio was significantly higher $(\mathrm{p}<0.05)$ in female fed STZ rats than in female fed normal rats. On the contrary, it was lower $(\mathrm{p}<0.01)$ in female GK rats $(1.13 \pm 0.01 ; n=4)$ than in female normal rats $(1.24 \pm 0.02 ; n=8)$. It was also significantly lower $(\mathrm{p}<0.005)$ in female GK rats than in male GK rats.

Four ratios were examined concerning the long-chain polyunsaturated $\omega 6$ fatty acids. Whenever calculable, the $\mathrm{C} 18: 2 \omega 6 / \mathrm{C} 18: 3 \omega 6$ ratio was decreased $(\mathrm{p}<0.01)$ after overnight fasting to $78.7 \pm 4.2 \%(n=5)$ of the mean corresponding value found in rats of the same strain and sex $(100.0 \pm 2.9 \% ; n=4)$. Likewise, it was significantly lower $(\mathrm{p}<0.01)$ in GK rats $(113.6 \pm 8.4 ; n=5)$ than in normal rats $(171.0 \pm 13.6 ; n=4)$. No C18:3 $\omega 6$ was be detected, however, in the STZ rats $(n=6)$.

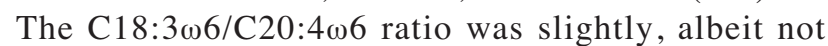
significantly, lower in overnight fasted normal female rats than in fed normal female rats. This ratio failed to differ significantly $(\mathrm{p}>0.3)$ in these normal animals $\left(2.72 \pm 0.12 \times 10^{-3}\right.$; $\mathrm{n}=4)$ and GK rats $\left(2.93 \pm 0.16 \times 10^{-3} ; \mathrm{n}=5\right)$.

The C18:2 $\omega 6 / \mathrm{C} 20: 4 \omega 6$ paired ratio in spleen phospholipids averaged, in overnight fasted normal and GK rats, respectively, $68.7 \pm 1.3 \%(n=4 ; p<0.001)$ and $81.0 \pm 2.0 \%$ $(\mathrm{n}=4 ; \mathrm{p}<0.001)$ of the mean corresponding value found in fed animals $(100.0 \pm 3.5 \%$ and $100.0 \pm 0.3 \% ; n=4$ in both cases $)$. It was decreased from $0.544 \pm 0.019(\mathrm{n}=4)$ in fed female normal rats to $0.473 \pm 0.028(n=6 ; p>0.1)$ in fed female STZ rats and $0.371 \pm 0.002(\mathrm{n}=2 ; \mathrm{p}<0.005)$ in fed female GK rats. It failed to differ significantly in either fed or fasted male versus female GK rats.

Lastly, in normal rats, the $C 20: 4 \omega 6 / C 22: 4 \omega 6$ ratio was increased by overnight fasting $(\mathrm{p}<0.005)$. Such an increase failed, however, to achieve statistical significance $(p>0.1)$ in
GK rats. The $\mathrm{C} 20: 4 \omega 6 / \mathrm{C} 22: 4 \omega 6$ paired ratio was significantly higher ( $\mathrm{p}<0.02$ or less) in fed female STZ rats and fed female GK rats than in fed female normal rats. It was also higher $(\mathrm{p}<0.025)$ in female GK rats $(11.65 \pm 0.35 ; \mathrm{n}=4)$ than in male GK rats $(10.49 \pm 0.13 ; n=4)$.

Next, three ratios were examined concerning the longchain polyunsaturated $\omega 3$ fatty acids. First, the C18:3 $03 /$ $\mathrm{C} 20: 5 \omega 3$ ratio was only established in 6 animals, with a mean value of $1.20 \pm 0.14$. Second, the $C 20: 5 \omega 3 / C 22: 5 \omega 3$ ratio was not significantly different in normal rats $\left(41.5 \pm 7.3 \times 10^{-3} ; \mathrm{n}=6\right)$, STZ rats $\left(73.2 \pm 14.7 \times 10^{-3} ; \mathrm{n}=4\right)$ and $\mathrm{GK}$ rats $\left(59.9 \pm 10.7 \times 10^{-3} ; \mathrm{n}=5\right)$. Third, the C22:5 $\mathrm{\omega} 3 / \mathrm{C} 22: 6 \omega 3$ ratio was not affected significantly $(p>0.1)$ by fasting with the results recorded in overnight fasted rats averaging $94.5 \pm 2.0 \%(n=8)$ of those found in fed animals of the same strain and sex $(100.0 \pm 2.6 \%$; $\mathrm{n}=8)$. It was decreased $(\mathrm{p}<0.025$ or less) from $0.833 \pm 0.039$ $(n=4)$ in fed female normal rats to $0.686 \pm 0.034(n=6)$ in fed female STZ rats and to $0.419 \pm 0.029(n=2)$ in fed female GK rats. This ratio was significantly lower $(\mathrm{p}<0.001)$ in female GK rats $(0.411 \pm 0.013 ; n=4)$ than in male GK rats $(0.631 \pm 0.019$; $n=4)$.

Triglycerides. The triglyceride content of the spleen, expressed as fatty acids, was not significantly different in the 5 groups of rats under consideration, with an overall mean value of $3.34 \pm 0.74 \mathrm{mg} / \mathrm{g}$ wet wt $(\mathrm{n}=22)$.

As indicated by the SEM mentioned in Table III, the total fatty acid content of spleen triglycerides was quite variable. Fig. 1 illustrates the inverse correlation between such a content and the weight percentage of C18:0.

The three major fatty acids found in the triglycerides were

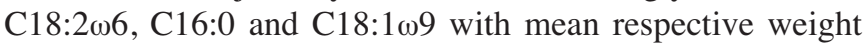
percentages of $38.8 \pm 0.9,25.3 \pm 0.6$ and $21.5 \pm 0.5 \%(n=22$ in all cases). Less abundant fatty acids identified in the spleen triglycerides included $(\mathrm{n}=22$ in all cases) $\mathrm{C} 14: 0(0.90 \pm 0.14 \%)$,

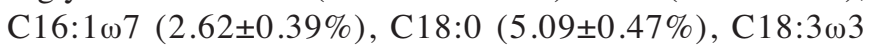




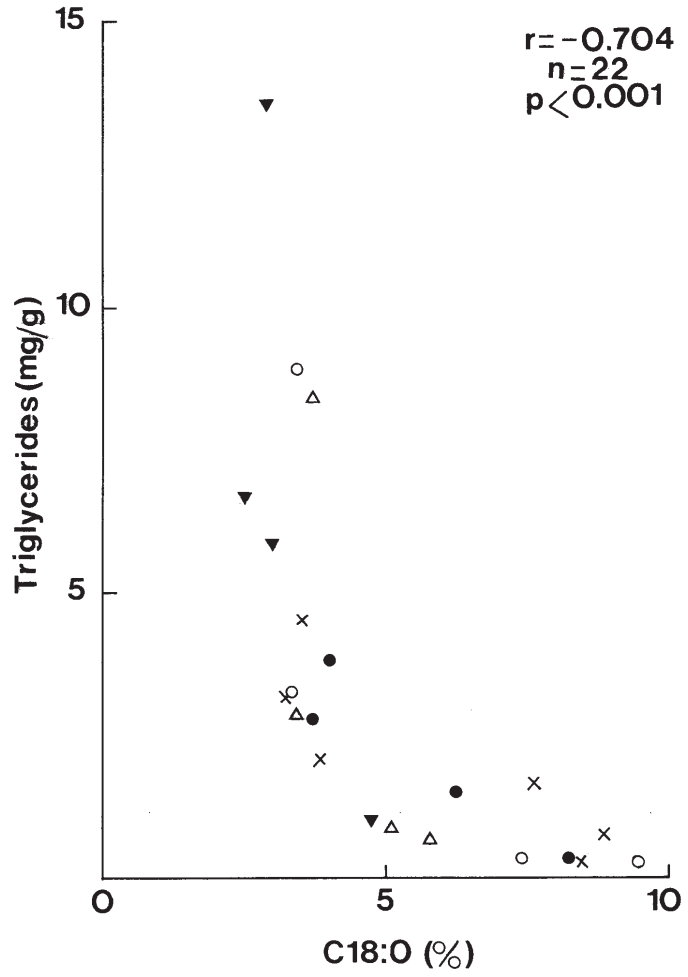

Figure 1. Relationship between the total amount of fatty acids in spleen triglycerides (mg/g wet wt) and their relative content in C18:0 (\%) in fed normal female rats $(\odot)$, overnight fasted normal female rats $(\bullet)$, fed STZ female rats $(\mathrm{x})$, GK female rats $(\Delta)$, and GK male rats $(\triangle)$.

$(1.86 \pm 0.26 \%), \mathrm{C} 22: 4 \omega 6(0.69 \pm 0.19 \%), \mathrm{C} 22: 5 \omega 3(0.84 \pm 0.25 \%)$ and $\mathrm{C} 22: 6 \omega 3(1.45 \pm 0.45 \%)$.

To note, C20:4w6 was undetected in the spleen triglycerides, except in 2 out of 22 rats, in which it represented $124 \pm 2 \mu \mathrm{g} / \mathrm{g}$ wet wt.

Significant differences for the weight percentage of certain fatty acids in distinct groups of rats were not exceptional, as illustrated by the three following examples. First, obvious gender differences were sometimes observed. For instance, the $\mathrm{C} 16: 1 \omega 7$ weight percentage was twice higher $(\mathrm{p}<0.005)$

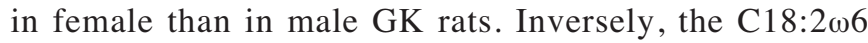
relative contribution was lower $(\mathrm{p}<0.005)$ in female than male GK rats (Table III). Second, fasting also affected the weight percentage of certain fatty acids. For instance, the relative contribution of $\mathrm{C} 18: 3 \mathrm{\omega} 3$ to the spleen triglycerides represented, after overnight fasting, 80.4 $4.3 \% \quad(n=6$; $\mathrm{p}<0.005$ ) of the paired value found in fed rats of the same sex and strain examined within the same experiment(s). Finally, a marked difference between normal and diabetic rats was also on occasion noticed. For instance, the relative contribution of $\mathrm{C} 22: 4 \omega 6, \mathrm{C} 22: 5 \omega 3$ and $\mathrm{C} 22: 6 \omega 3$ were in fed STZ and GK rats, respectively, $7.10 \pm 2.10(n=18 ; \mathrm{p}<0.01)$ and $5.43 \pm 1.05(n=6 ; p<0.01)$ times higher than that found in fed normal rats of the same sex. Likewise, in overnight fasted rats, the relative contribution of these three fatty acids was $2.61 \pm 0.15$ times higher $(n=6 ; p<0.001)$ in female GK rats than in female normal animals.

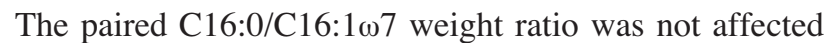
significantly by fasting. It averaged, in female rats, $7.98 \pm 0.86(n=5)$, in normal rats, $6.41 \pm 0.34(n=3)$, in STZ rats, and $5.50 \pm 0.64(\mathrm{n}=4)$, in GK rats, none of these values being significantly different from one another. It was higher $(\mathrm{p}<0.005)$, however, in the male GK rats $(11.24 \pm 1.06 ; n=4)$ than in the female GK rats.

The C18:0/C18:1 19 ratio was not significantly different in the female fed or fasted normal rats and fed STZ rats, with an overall mean value of $0.297 \pm 0.040(n=14)$. It was lower in male GK rats $(0.203 \pm 0.029 ; \mathrm{n}=4)$ and, even more so, in female GK rats $(0.134 \pm 0.022 ; n=4)$, the difference between these type-2 diabetic rats and the type-1 diabetic STZ rats achieving statistical significance $(\mathrm{p}<0.05)$.

The $(\mathrm{C} 16: 0+\mathrm{C} 16: 1 \omega 7) /(\mathrm{C} 18: 0+\mathrm{C} 18: 1 \omega 9)$ ratio was also comparable in the female fed or fasted normal rats and fed STZ rats, with an overall mean value of $1.081 \pm 0.028(n=14)$. Virtually the same mean value $(1.086 \pm 0.045 ; n=4)$ was found in female fed and fasted GK rats. The latter value differed $(\mathrm{p}<0.05)$, however, from that found in male GK rats $(0.912 \pm 0.043 ; n=4)$.

The $\mathrm{C} 18: 2 \omega 6 / \mathrm{C} 18: 3 \omega 6$ ratio could be assessed in only a few rats. It averaged $220 \pm 6$ in three female normal rats, as distinct $(\mathrm{p}<0.001)$ from no more than $134 \pm 5$ in three female GK rats. The $\mathrm{C} 18: 2 \omega 6 / \mathrm{C} 20: 4 \omega 6$ ratio was calculable in only two rats $(15.8 \pm 1.7 ; n=2)$. The $\mathrm{C} 18: 2 \omega 6 / \mathrm{C} 22: 4 \omega 6$ ratio averaged $133 \pm 12$ in four normal rats, as distinct $(\mathrm{p}<0.02$ or less) from $38 \pm 23$ in three STZ rats and $56 \pm 13$ in eight GK rats. Thus, the restricted information on the content of spleen triglycerides in long-chain polyunsaturated $\omega 6$ fatty acids suggested a more efficient metabolism of these fatty acids in diabetic rats than in control animals.

Finally, as far as the long-chain polyunsaturated $\omega 3$ fatty acids are concerned, the $\mathrm{C} 18: 3 \omega 3 / \mathrm{C} 22: 5 \omega 3$ ratio was also higher $(\mathrm{p}<0.01)$ in normal rats $(7.62 \pm 0.81 ; \mathrm{n}=4)$ than in the STZ and GK diabetic rats $(3.70 \pm 0.77 ; n=8)$. The $C 22: 5 \omega 3$ / C22:6w3 ratio, however, was not significantly different in female normal rats $(0.509 \pm 0.049 ; \mathrm{n}=4)$, STZ rats $(0.454 \pm 0.034 ; n=3)$ and GK rats $(0.377 \pm 0.060 ; n=4)$. The sole significant difference $(\mathrm{p}<0.01)$ concerned the higher value found in male GK rats $(0.782 \pm 0.082 ; n=3)$ than in female GK rats or other female animals.

\section{Discussion}

The present study affords three major pieces of information. First, a comparison of data collected in such organs as the liver, skeletal muscle, heart and brain, documents dramatic differences in the fatty acid content and pattern of both phospholipids and triglycerides. To cite only one example and to restrict the comparison to the spleen versus the liver of normal rats, it reveals that, in addition to a twice lower total content of fatty acids in spleen phospholipids, their C22:6w3 relative content is also 4 times lower than in the liver (5). Likewise and also in addition to a total fatty acid content of triglycerides almost twice lower in the spleen, their C18:2w6 relative content averaged $39.7 \pm 1.9 \%$ as distinct $(\mathrm{p}<0.005)$ from $22.9 \pm 2.1 \%$ in the liver of the same animals. In several organs, e.g. in muscle (6), a significant negative correlation prevails between the total fatty acid content of triglycerides

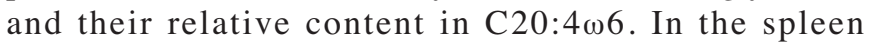
triglycerides, a comparable inverse relationship was found when considering the weight percentage of C18:0. 

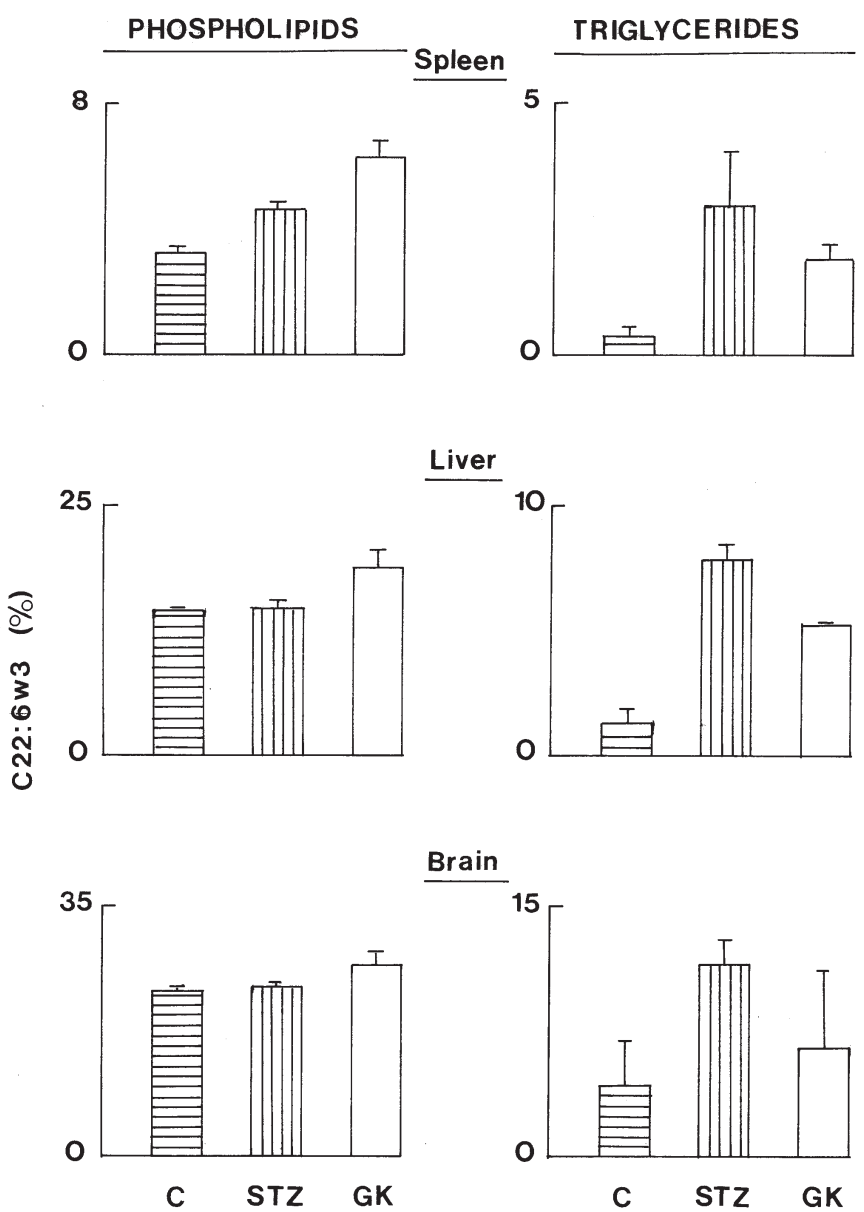

Brain

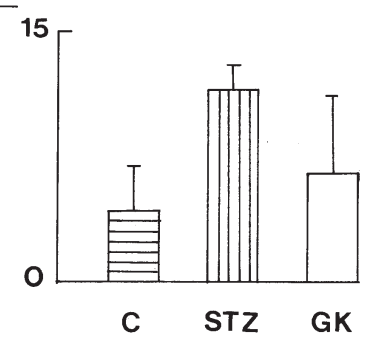

Figure 2. Weight percentage of $\mathrm{C} 22: 6 \omega 3$ in the phospholipids (left) and triglycerides (right) of the spleen (upper panels), liver (middle panels) and brain (lower panels) of fed female normal rats (horizontally hatched column), STZ rats (vertically hatched columns) and GK rats (open columns). Mean values ( \pm SEM) refer to 2-6 animals.

Second, the present study extends to the spleen the knowledge that the fatty acid pattern of both phospholipids and triglycerides may be affected by such factors as gender, nutritional status (fed versus overnight fasted rats) and metabolic status (control versus diabetic rats). Moreover, selected lipid variables were significantly different in the animal models of type- 1 and type- 2 diabetes used in the present study.

Third, focus must be drawn to specific diabetes-related changes found in the spleen, as distinct from other organs, e.g. the liver or the brain $(3,5)$. In this respect, the following illustrative finding merits attention (Fig. 2). In the spleen phospholipids, the weight percentage of $\mathrm{C} 22: 6 \mathrm{w} 3$ found in fed female rats was significantly higher in STZ rats than in control animals $(\mathrm{p}<0.005)$ and in GK rats than STZ rats $(\mathrm{p}<0.02)$. In the liver phospholipids, however, a significant increase $(\mathrm{p}<0.05$ or less) in the weight percentage of $\mathrm{C} 22: 6 \omega 3$ above the value found in control animals was only observed in GK rats, whether the comparison between the female control animals and female GK rats referred to fed or fasted rats. Lastly, in brain phospholipids of female GK rats, a significant increase in the weight percentage of C22:6 33 above the value found in female normal rats was only reached by pooling the results obtained in fed and fasted animals, the measurements made in GK rats averaging $112.1 \pm 4.0 \% \quad(n=4$; $\mathrm{p}<0.01$ ) of the mean corresponding values found in normal rats examined in the same nutritional state $(100.0 \pm 1.7 \%$; $\mathrm{n}=8$ ). In the case of the fed female GK rats, the results recorded in the spleen, liver and brain represented, respectively, $190.4 \pm 13.9 \%(\mathrm{p}<0.001), 131.0 \pm 12.5 \%(\mathrm{p}<0.05)$ and $116.2 \pm 8.0 \%(\mathrm{p}>0.05)$ of the mean corresponding values found in fed female normal rats. The latter values clearly document the vastly different relative magnitude of the diabetesinduced changes (rank correlation coefficient: 0.887 ; $n=6$; $\mathrm{p}<0.02$ ) in the three organs under consideration. In sharp contrast to this situation, the pattern of the diabetes-related changes in the relative contribution of $\mathrm{C} 22: 6 \omega 3$ to triglycerides was comparable in the spleen, liver and brain (Fig. 2, right panel). Indeed, whether in the spleen, liver or brain, the mean weight percentage of $\mathrm{C} 22: 6 \mathrm{w} 3$ in triglycerides was always higher in GK rats than in control animals and always further increased in STZ rats. For instance, relative to the fractional contribution of C22:6w3 found in the triglycerides of STZ rats, that recorded in GK rats was comparable in the spleen, liver and brain, with an overall mean value of $59.8 \pm 11.3 \%$ $(\mathrm{n}=6 ; \mathrm{p}<0.02$ versus unity). The latter difference and its similarity in distinct organs may well reflect the invariable participation of metabolic or hormonal factors to the regulation of fatty acid de novo synthesis and their further incorporation in the triglycerides of these organs. In this respect, the severe hypoinsulinemia of STZ rats contrasting with normal to elevated plasma insulin concentrations in GK rats may well represent a major regulatory factor. In the case of phospholipids, however, the vastly different pattern of the diabetes-related changes in their fatty acid pattern, as observed in distinct organs, argues in favour of an adaptive process dictated by the specific function of each organ.

Distinct regulatory influences in phospholipid versus triglyceride formation are also supported by comparison of the paired ratio between distinct fatty acids in these two classes of lipids found in different types of rats. For instance, in the triglycerides, the $\mathrm{C} 18: 0 / \mathrm{C} 18: 1 \omega 9$ ratio was always lower in fed female GK rats than in fed female STZ rats, whether in the spleen, liver or brain. In these three organs, such a ratio averaged, in the GK rats, $59.9 \pm 10.1 \% \quad(n=6$; $\mathrm{p}<0.04$ ) of the mean corresponding values found in STZ rats $(100.0 \pm 9.5 \% ; n=18)$, suggesting higher $\omega 9$-desaturase activity in the former than latter rats. In the case of the phospholipids, however, the $\mathrm{C} 18: 0 / \mathrm{C} 18: 1 \omega 9$ ratio was never significantly different in fed female GK and STZ rats. The measurements collected in GK rats indeed averaged $109.6 \pm 9.0 \%(n=5 ; p>0.2)$ of the mean corresponding values found in the spleen, liver and brain of STZ rats $(100.0 \pm 3.0 \%$; $\mathrm{n}=18$ ).

In conclusion, the results of the present study not only document differences in the phospholipid and triglyceride fatty acid pattern of spleen lipids as compared to either liver or brain lipids, but also extend our knowledge of the spleen to include the fact that such a fatty acid pattern is regulated by nutritional and hormonal factors, as illustrated by differences between fed and fasted rats, normal and diabetic rats, or male and female animals. Finally, and most notedly, the results reveal, by comparison with data collected in the liver 
and brain of the same animals, independent regulation of triglyceride and phospholipid formation. Thus, on one hand, the fatty acid pattern of triglycerides and its alteration in type- 1 or type- 2 diabetes are closely similar in the spleen, liver and brain, pointing to a common regulatory factor such as plasma insulin concentration. On the other hand, however, certain variables of the fatty acid pattern, which are obviously affected to a different extent in distinct animal models of diabetes at the level of tissue triglycerides, may fail to display any significant difference in these diabetic animals at the level of tissue phospholipids. Likewise, in the case of the C22:6 63 weight percentage, the diabetes-induced changes are comparable in the triglycerides of the spleen and the brain, but vastly different in the phospholipids of these three organs. It is proposed that these observations point to a tissue-specific adaptative regulation of phospholipid formation, possibly linked to the function of each organ. For instance, that the diabetes-induced changes in the C22:6w3 relative content of phospholipids were more pronounced in the spleen than in either the liver or the brain, could reflect a major role of this long-chain polyunsaturated $\omega 3$ fatty acid in the defence mechanisms insured by splenocytes and their adaptation to diabetic stress.

\section{Acknowledgements}

This study was supported in part by a grant from the Région de Bruxelles-Capitale (Convention RBC-BR 246/2410) to Target Hit s.a. (Brussels, Belgium). We are grateful to A. Chwalik and A. Dufour for technical assistance and C. Demesmaeker for secretarial help.

\section{References}

1. Malaisse WJ, Portois L, Zhang Y, Oguzhan B, Louchami K, Jijakli H, Courtois P, Sener A and Carpentier YA: Fatty acid content and pattern of edipidymal and parametrial adipose tissue lipids in streptozotocin (type 1) and Goto-Kakizaki (type 2) diabetic rats. Int J Mol Med 18: 1231-1234, 2006.

2. Carpentier YA, Fontaine D, Otto A, Portois L, Fontaine $\mathrm{J}$ and Malaisse WJ: Sustained enrichment of liver phospholipids and triglycerides in eicosapentaenoate after a bolus intravenous injection of a medium-chain triglycerides:fish oil emulsion to streptozotocin (Type 1) and Goto-Kakizaki (Type 2) diabetic rats. Int J Mol Med 17: 643-647, 2006.

3. Malaisse WJ, Zhang Y, Louchami K, Sener A, Portois L and Carpentier YA: Brain phospholipid and triglyceride content and fatty acid pattern in type 1 and type 2 diabetic rats. Abstracts 88th Annual Meeting of the Endocrine Society 238-239, 2006.

4. Richelle M, Carpentier YA and Deckelbaum RJ: Long- and medium-chain triacylglycerols in neutral lipid-exchange processes with human plasma low-density lipoproteins. Biochemistry 33: 4872-4878, 1984.

5. Malaisse WJ, Portois L, Sener A and Carpentier YA: Fatty acid pattern of plasma and tissue lipids in two animal models of diabetes mellitus. Int J Mol Med 18: (Suppl), Abst., 2006. 